Cross Section Evaluations for Arsenic Isotopes

J. Pruet, D. P. McNabb, W. E. Ormand

March 14, 2005 
This document was prepared as an account of work sponsored by an agency of the United States Government. Neither the United States Government nor the University of California nor any of their employees, makes any warranty, express or implied, or assumes any legal liability or responsibility for the accuracy, completeness, or usefulness of any information, apparatus, product, or process disclosed, or represents that its use would not infringe privately owned rights. Reference herein to any specific commercial product, process, or service by trade name, trademark, manufacturer, or otherwise, does not necessarily constitute or imply its endorsement, recommendation, or favoring by the United States Government or the University of California. The views and opinions of authors expressed herein do not necessarily state or reflect those of the United States Government or the University of California, and shall not be used for advertising or product endorsement purposes.

This work was performed under the auspices of the U.S. Department of Energy by University of California, Lawrence Livermore National Laboratory under Contract W-7405-Eng-48. 


\title{
Cross Section Evaluations for Arsenic Isotopes
}

\author{
J.Pruet, D.P. McNabb \& W.E.Ormand
}

\begin{abstract}
We present an evaluation of cross sections describing reactions with neutrons incident on the arsenic isotopes with mass numbers 75 and 74 . Particular attention is paid to $(\mathrm{n}, 2 \mathrm{n})$ reactions. The evaluation for ${ }^{75} \mathrm{As}$, the only stable As isotope, is guided largely by experimental data. Evaluation for ${ }^{74} \mathrm{As}$ is made through calculations with the EMPIRE statistical-model reaction code. Cross sections describing the production and destruction of the $26.8 \mathrm{~ns}$ isomer in ${ }^{74} \mathrm{As}$ are explicitly considered. Uncertainties and covariances in some evaluated cross sections are also estimated.
\end{abstract}

\section{Introduction}

Here we document a new evaluation of some arsenic cross sections. This effort has been made because LLNL's ENDL evaluations for As are undocumented and predate modern statistical model calculations as well as some important experiments. In particular, Walid Younes and his collaborators at LLNL have recently performed high quality measurements of the ${ }^{75} \mathrm{As}(\mathrm{n}, 2 \mathrm{n})^{74 \mathrm{~m}}$ As reaction cross section (Younes et al 2003). This recent experiment allows the first careful evaluation of the isomeric production cross section.

Another motivation for this effort relates to recent interest in implications of nuclear data uncertainties. Traditionally, data uncertainties and covariances have received little attention in evaluations. In fact, LLNL's ENDL format has only recently been extended to represent uncertainties. As part of this evaluation we present covariance matrices as well as some discussion about how these matrices are estimated and implemented.

Experimental data is used in this evaluation whenever possible. This choice is made because uncertainties in measured cross sections are often appreciably smaller than uncertainties in calculated cross sections. Cross sections that have not been well measured are calculated using the statistical-model reaction code EMPIRE (Herman 2002). In some cases experiments are used to suggest choices for level densities and optical model potentials used in calculations.

Experimental data used in this evaluation was mined from the EXFOR repository and from Younes et al (2003). In general it is necessary to check original literature referred to by 
EXFOR entries. The quality of a given experiment and the proper interpretation of listed uncertainties are necessarily not conveyed in the terse EXFOR format.

Fits to experimental data are made using splines as basis functions through the code da_fit written by David Brown of LLNL. Though splines would not be a good choice for describing resonances, they are expected to do a fair job of representing, e.g., smoothly varying $(n, 2 n)$ cross sections. Formally, these spline fits could also be used to define covariance matrices. As this would be poorly motivated from a physical standpoint, we describe in section 3.4 estimates of covariances based on statistical-model reaction calculations.

\section{Evaluation for $\mathbf{n}+{ }^{75} \mathrm{As}$}

A number of experiments have studied reactions involving this only stable isotope of arsenic. In addition, there is a complete JENDL evaluation for $n+{ }^{75}$ As. The JENDL evaluation is based mostly on statistical-model calculations made with the EGNASH code. Our approach combines experimental data with model calculations.

\section{1. ${ }^{75} \mathrm{As}(\mathbf{n}, 2 \mathbf{n}){ }^{74} \mathrm{As}$}

We present evaluations for the total $(n, 2 n)$ cross section as well as for the partial cross sections leading to the production of the ground and meta-stable states in ${ }^{74}$ As. The metastable state in ${ }^{74} \mathrm{As}$ is assigned as $J^{\pi}=(4)^{+}$, lies $259.29 \mathrm{keV}$ above the ground state and has half-life $\tau=26.8 \mathrm{~ns}$. Since the isomer decays far faster than weak-decay timescales, every ${ }^{75} \mathrm{As}(\mathrm{n}, 2 \mathrm{n})$ reaction eventually leads to population of the ${ }^{74}$ As ground state. By definition, the ground state production cross section is that part of the $(n, 2 n)$ cross section which does not lead to population of ${ }^{74 m}$ As. With this convention,

$$
\sigma_{\mathrm{n}, 2 \mathrm{n}}^{(t o t)}=\sigma_{\mathrm{n}, 2 \mathrm{n}}^{(m)}+\sigma_{\mathrm{n}, 2 \mathrm{n}}^{(g)}
$$

where (tot), (g), and (m) refer to the total, ground state production, and isomeric production cross sections.

Experimental and evaluated data for the total $(n, 2 n)$ cross section is shown in figure 1. The spline fit to the data is characterized by $\chi^{2}=1.06$ per degree of freedom, indicating that the fit is in good agreement with data. About a dozen experiments have studied $\sigma_{\mathrm{n}, 2 \mathrm{n}}^{(t o t)}$, with most available data describing this reaction at incident neutron energies $E_{n}$ near 14 $\mathrm{MeV}$. Overall there is good agreement between the present evaluation, the previous ENDL evaluation, and the JENDL-3.3 evaluation. 
The $(\mathrm{n}, 2 \mathrm{n})$ cross section near threshold needs special attention. Only two experiments (Prestwood \& Bayhurst 1961; Birn \& Qaim 1994) provide data for this cross section at incident energies less than 12.2 MeV. Uncertainties given by Prestwood \& Bayhurst (1961) "were those which gave the best fit of the data to a theoretical excitation function" and are typically about $5 \%$. This uncertainty is not a true experimental error and is remarkably low compared to uncertainties present in other experiments. We have set all uncertainties in the Prestwood \& Bayhurst (1961) data to $20 \%$, which is roughly comparable to uncertainties in similar $(\mathrm{n}, 2 \mathrm{n})$ cross section measurements made in the late 1950's. Uncertainties given by Birn \& Qaim (1994) seem to have been carefully derived. However, that experiment reports $\mathrm{a} \sim 250 \mathrm{keV}$ uncertainty in the incident neutron energy. As our fitting code does not account for uncertainties in the incident neutron energy, we have approximately translated the uncertainty in incident energies into uncertainties in the cross section at a fixed energy:

$$
\delta_{\text {eff }}^{2}(\sigma) \equiv \delta_{\text {expt }}^{2}(\sigma)+\left(\frac{d \sigma}{d E_{n}} \delta_{\text {expt }}\left(E_{n}\right)\right)^{2} .
$$

Here $\delta(X)$ represents the standard error in the quantity $X, \delta_{\text {expt }}$ represents the stated experimental uncertainty, and $d \sigma / d E_{n}$ represents the slope of the cross section. At $12 \mathrm{MeV}$, $d \sigma / d E_{n} \approx 400 \mathrm{mb} / \mathrm{MeV}$, so that an uncertainty of $250 \mathrm{keV}$ in the incident energy implies an additional uncertainty in the cross section of about $100 \mathrm{mb}$.

The slope of the $(n, 2 n)$ cross section at threshold is expected to be identically zero. To account for this we have scaled the spline fits to the data. This scaling is only made for incident neutron energies lower than are described by experimental data and is done so that i) the slope and value of the evaluated cross section at threshold are both zero, and ii) the slope and value of the evaluated cross section are continuous.

\section{2. $\quad{ }^{75} \mathrm{As}(\mathbf{n}, 2 \mathbf{n}){ }^{74 m} \mathrm{As}$}

Figure 2 shows experimental data and the present evaluation for the ${ }^{75} \mathrm{As}(\mathrm{n}, 2 \mathrm{n}){ }^{74 m}$ As cross section. Apart from the LLNL experiment only one other experiment has addressed production of the isomer in ${ }^{74}$ As (Sothras 1977). A reading of Sothras' thesis revealed that the lifetime of ${ }^{74 m}$ As was misidentified as $8 \mathrm{sec}$. Because Sothras used a cyclic activation/counting technique which relies on accurately knowing half-lives, we have neglected the Sothras datum. 


\section{3. $\quad{ }^{75} \mathrm{As}(\mathbf{n}, 2 \mathbf{n}){ }^{74 g} \mathrm{As}$}

The ground state production cross section is defined as the difference of the total and isomeric production cross sections. The ground state production cross section accounts for about $70 \%$ of the $(n, 2 n)$ reaction cross section for incident neutron energies near $14 \mathrm{MeV}$.

\section{4. $\quad{ }^{75} \operatorname{As}(\mathrm{n}$, tot $)$}

Figure 3 shows EMPIRE calculations, experimental data, and evaluations describing the total $n+{ }^{75}$ As cross section. All EMPIRE calculations discussed in this paper make use of the global optical model potential for neutrons described by Koning \& Delaroche (2003). Our evaluation for $\sigma$ (n,tot) uses the EMPIRE calculations for $E_{n}<2.27 \mathrm{MeV}$ and $E_{n}>14.85 \mathrm{MeV}$. Zucker (1956) addresses the total cross section at low incident neutron energies $0.1<E_{n}<2 \mathrm{MeV}$. However, because uncertainties are not given for this experiment - and because these uncertainties appear from the data to be appreciable - we have neglected the Zucker (1956) experiment.

\subsection{Other $\mathbf{n}+{ }^{75}$ As cross sections}

At low incident neutron energies a modest array of experiments have addressed different neutron reactions for ${ }^{75}$ As. At higher incident energies experimental data is sparse except for the $(n, 2 n)$ and $(n, t o t)$ reactions. Above the $(n, 2 n)$ threshold there are only about five experimental data points for all other reactions combined.

Our evaluation for reactions other than $(n, 2 n)$ and $(n, t o t)$ is based in EMPIRE calculations. We have uniformly re-scaled cross sections other than $(n, 2 n)$ so that the sum of the different partial cross sections gives the evaluated total cross section. This re-scaling is modest $(\sim 4 \%)$. Figure 4 shows our evaluations for a few dominant reaction sections.

\section{Evaluation for $\mathbf{n}+{ }^{74} \mathrm{As}$}

Apart from ENDL no evaluations describe reactions with ${ }^{74} \mathrm{As}$ as a target. The ENDL evaluation is undocumented and appears to have been guided by systematics of reactions involving nearby stable nuclei. Because ${ }^{74}$ As is short-lived ( $\tau=17.77$ days) experiments have not used this nuclide as a target. It is possible that the planned RIA facility could be used to study reactions on ${ }^{74} \mathrm{As}$. 
The present evaluation for $n+{ }^{74}$ As is based entirely on EMPIRE calculations. A basic difficulty with this approach is that seemingly reasonable changes to input parameters cause appreciable changes in calculated results. For example, a look at calculations for many different isotopes shows that the Gilbert Cameron, Hartree-Fock BCS and EMPIRE-specific prescriptions for level densities reproduce experimental $(n, 2 n)$ cross sections with comparable accuracy. Without experimental information, then, there is no compelling reason to choose one prescription over the other. However, for a given isotope changing the choice of level density can change the $(n, 2 n)$ reaction cross section by as much as $20-30 \%$. As we discuss below, our present evaluation attempts to incorporate this uncertainty in the choice of input parameters.

\section{1. $\quad{ }^{74 g} \mathrm{As}(\mathbf{n}, 2 \mathbf{n}){ }^{73} \mathbf{A s}$}

Figure 5 shows EMPIRE calculations, the ENDL evaluation, and the present evaluation for the ${ }^{74 g} \mathrm{As}(\mathrm{n}, 2 \mathrm{n})$ reaction cross section. Different EMPIRE calculations shown here correspond to different level density parameterizations. Other changes to the input parameters - such as changing the prescription for pre-equilibrium, the optical model parameters, or the electromagnetic strength function - make small differences compared to the influence of changing the level density prescription. Because of the absence of experimental information for reactions involving ${ }^{74}$ As as a target, there doesn't seem to be a strong a-priori reason to choose one level density prescription over another.

Our evaluation uses the average of the three different EMPIRE calculations. Uncertainties in this evaluation are defined by the spread in results of the EMPIRE calculations. This decision cannot be rigorously justified. In general one would define an application-specific average. For example, suppose we had to do simulations for a particular reactor characterized by some neutron flux $\phi(E)$. In this case is might be appropriate to define an evaluated cross section based on a convolution of the cross section with $\phi(E)$. Simply averaging the cross section seems the best application-unspecific decision. Also, figure 5 shows that the different cross sections have similar shapes. This implies that sensitivity of an arbitrary system (or response to an arbitrary flux $\phi$ ) will be correctly reproduced as long as the covariances discussed in section 3.4 are implemented.

There is fair agreement between the present evaluation and the previous ENDL evaluation for the $(\mathrm{n}, 2 \mathrm{n})$ cross section at incident neutron energies larger than about $12.5 \mathrm{MeV}$. At lower incident neutron energies the ENDL evaluation rises unphysically sharply. For incident neutron energies smaller than $11 \mathrm{MeV}$ ENDL appears to systematically overestimate the $(n, 2 n)$ reaction cross section by about a factor of two. 


\section{2. ${ }^{74 m} \mathrm{As}(\mathbf{n}, \mathbf{2 n})^{73} \mathrm{As}$}

Figure 6 shows the EMPIRE calculation of the cross section for depletion of the isomer in ${ }^{74}$ As. This isomer has an unknown spin but is tentatively assigned as $J^{\pi}=(4)^{+}$. The ground state of ${ }^{74}$ As has $J^{\pi}=2^{-}$. Figure 6 shows that the difference between the ground and isomeric-state spins seems to have little influence on the $(n, 2 n)$ cross section relative to the influence of model uncertainties.

\subsection{Other $\mathbf{n}+{ }^{74}$ As cross sections}

As with the cross section for the $(\mathrm{n}, 2 \mathrm{n})$ reaction, all cross sections for ${ }^{74}$ As are based on EMPIRE calculations. We have again uniformly re-scaled cross sections other than (n,2n) so that the sum of the different partial cross sections gives the evaluated total cross section. Figure 7 shows our evaluations for cross sections describing a few important reactions.

\subsection{Estimates of covariance matrices}

In the previous sections we discussed assignment of uncertainties in evaluated cross sections. For experimentally-based evaluations these uncertainties arise directly from a combination of uncertainties in the different experiments. For the EMPIRE based evaluation of ${ }^{74} \operatorname{As}(n, 2 n)$ an uncertainty was assigned based on plausible values of the model input parameters. The basic motivation for including these error estimates is to allow computational modelers to quantify the influence of nuclear-data uncertainties on the performance of complicated systems. To do this, not only error estimates, but also estimates of covariance matrices, are needed.

A covariance matrix specifies how related quantities are to be sampled. Formally, the covariance between a random variable $x_{1}$ and a random variable $x_{2}$ is defined by

$$
\operatorname{cov}\left(x_{1}, x_{2}\right)=\left\langle\left(x_{1}-\bar{x}_{1}\right)\left(x_{2}-\bar{x}_{2}\right)\right\rangle,
$$

with angle brackets denoting an average and $\bar{x}$ denoting the mean of $x$. To illustrate use of eq. 3, suppose that two random variables are related:

$$
x_{1}=z ; x_{2}=4 z
$$

where $z$ is a random variable of mean zero and unit standard deviation. For this toy example $\operatorname{cov}\left(x_{1}, x_{2}\right)=\left\langle 4 z_{1}^{2}\right\rangle=4$. Clearly, if in a simulation one supposes that $x_{1}=0.1$, then one 
should also set $x_{2}=0.4$. In the general case, variables $\mathbf{x}=\left(x_{1}, x_{2}, x_{3}, ..\right)$ related by a covariance matrix are consistently sampled by setting

$$
\mathbf{x}=\overline{\mathbf{x}}+A^{T} \mathbf{z}
$$

Here $\overline{\mathbf{x}}$ is a vector containing the mean values of $\mathbf{x}$ and $\mathbf{z}$ is a vector of independently chosen random variables, each with mean zero and unit standard deviation. The matrix $A^{T}$ appearing in eq. 5 is triangular and comes from the so-called Chodolsky decomposition of the covariance matrix:

$$
V=A^{T} A
$$

with $V_{i j}=\operatorname{cov}\left(x_{i}, x_{j}\right)$ the covariance matrix.

It is clear from figure 5 that a given $(n, 2 n)$ cross section is strongly correlated over small energy scales. If in a simulation one sets the $(\mathrm{n}, 2 \mathrm{n})$ cross section at $14 \mathrm{MeV}$ to $\bar{\sigma}(14)+\delta \sigma(14)=1.24$ barns, it would clearly be incorrect to set the cross section at 14.1 $\mathrm{MeV}$ to $\bar{\sigma}(14.1)-\delta \sigma(14.1)=1.08$ barns. To be more definite, we take the view that a cross section is a function of poorly defined model input parameters (e.g. the choice of level density). In this case the covariance matrix and correlation length can be estimated directly from calculations. In the specific cases of the $(n, 2 n)$ reaction cross sections presented above, we take the different EMPIRE calculations, as well as the present evaluations, to directly compute an estimate for the covariance matrix in eq. 3 .

For illustration we show in figure 8 the current evaluation for $\operatorname{cov}\left(\sigma(E), \sigma\left(E^{\prime}\right)\right)$ for the ${ }^{74} \mathrm{As}(\mathrm{n}, 2 \mathrm{n})$ reaction cross section. There is a marked correlation in the cross section values when $E$ and $E^{\prime}$ are both less than about $18 \mathrm{MeV}$. The influence of uncertainties in the model parameters going into the EMPIRE calculations seems to be just an overall scale factor at incident energies less than about $18 \mathrm{MeV}$. An estimate of the covariance matrix for the ${ }^{75} \mathrm{As}(\mathrm{n}, 2 \mathrm{n})$ reaction cross section is also included as part of this evaluation.

\section{Summary}

A complete new evaluation of cross sections for ${ }^{75} \mathrm{As}$ and ${ }^{74} \mathrm{As}$ has been presented. This evaluation includes estimates of covariances and uncertainties for $(n, 2 n)$ reaction cross sections. Comparisons with previous evaluations are favorable except for ${ }^{74} \mathrm{As}(\mathrm{n}, 2 \mathrm{n})$. For this reaction the previous ENDL evaluation appears to have appreciably over-estimated the cross section at low incident neutron energies.

This work was performed under the auspices of the US Department of Energy by Lawrence Livermore National Laboratory under contract W-7405-ENG-48. 


\section{REFERENCES}

Birn, I. \& Qaim, S. M. 1994, Nucl. Sci. and Eng. 116, 125

Foster, D.G. \& Glasgow, D.W. 1971, Phys. Rev. C 3, 576

Herman, M. 2002, "EMPIRE-II statistical model code for nuclear reaction calculations", available from the NNDC

Hsu, Y. C., Huang, C. Y., Tseng, P. K. \& Tai, Y. K. 1963, Chinese Jo. of Phys. 1, 6304

Koning, A.J. \& Delaroche, J.P. (2003), Nucl. Phys. A 713, 231

Manero, F. 1967, Nucl. Phys. 65, 419

Prestwood, R. J. \& Bayhurst, B. P. 1961, Phys. Rev. 121, 1438

Sothras, T. 1977, "A study of the systematics for $(\mathrm{n}, 2 \mathrm{n})$ reactions", dissertation thesis, Dissertation Abstracts B, 38, 280

Younes, W. et al., LLNL internal report, UCRL-ID-154061, 2003

Zucker, M.S. 1956, Phys. Rev. 104, 1025 


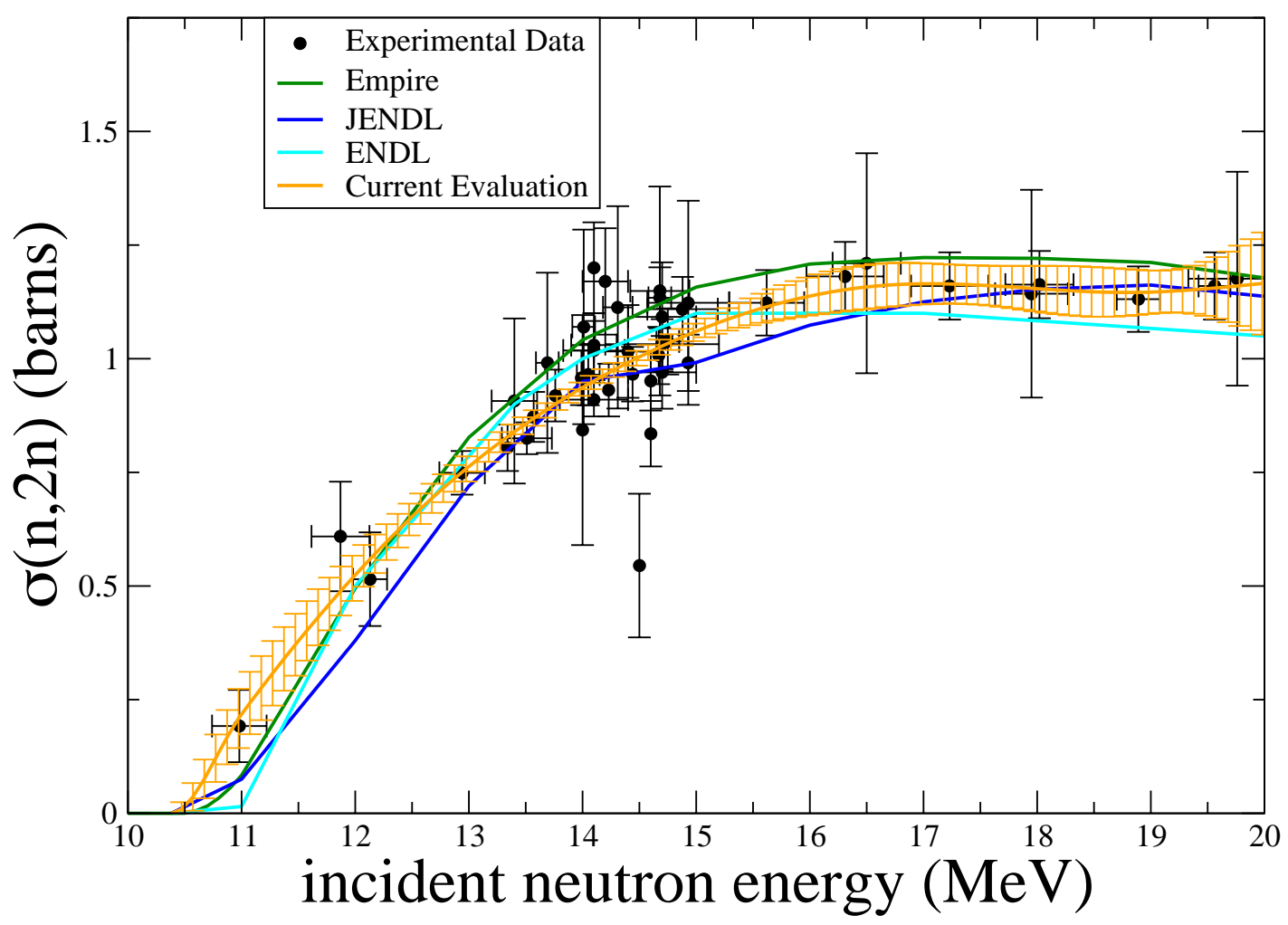

Fig. 1. - Data and evaluations for the total ${ }^{75} \mathrm{As}(\mathrm{n}, 2 \mathrm{n})$ cross section. Overall our data-based evaluation is in good agreement with the computationally-based JENDL evaluation as well as the ENDL evaluation. Also shown in this figure are results from EMPIRE calculations. 


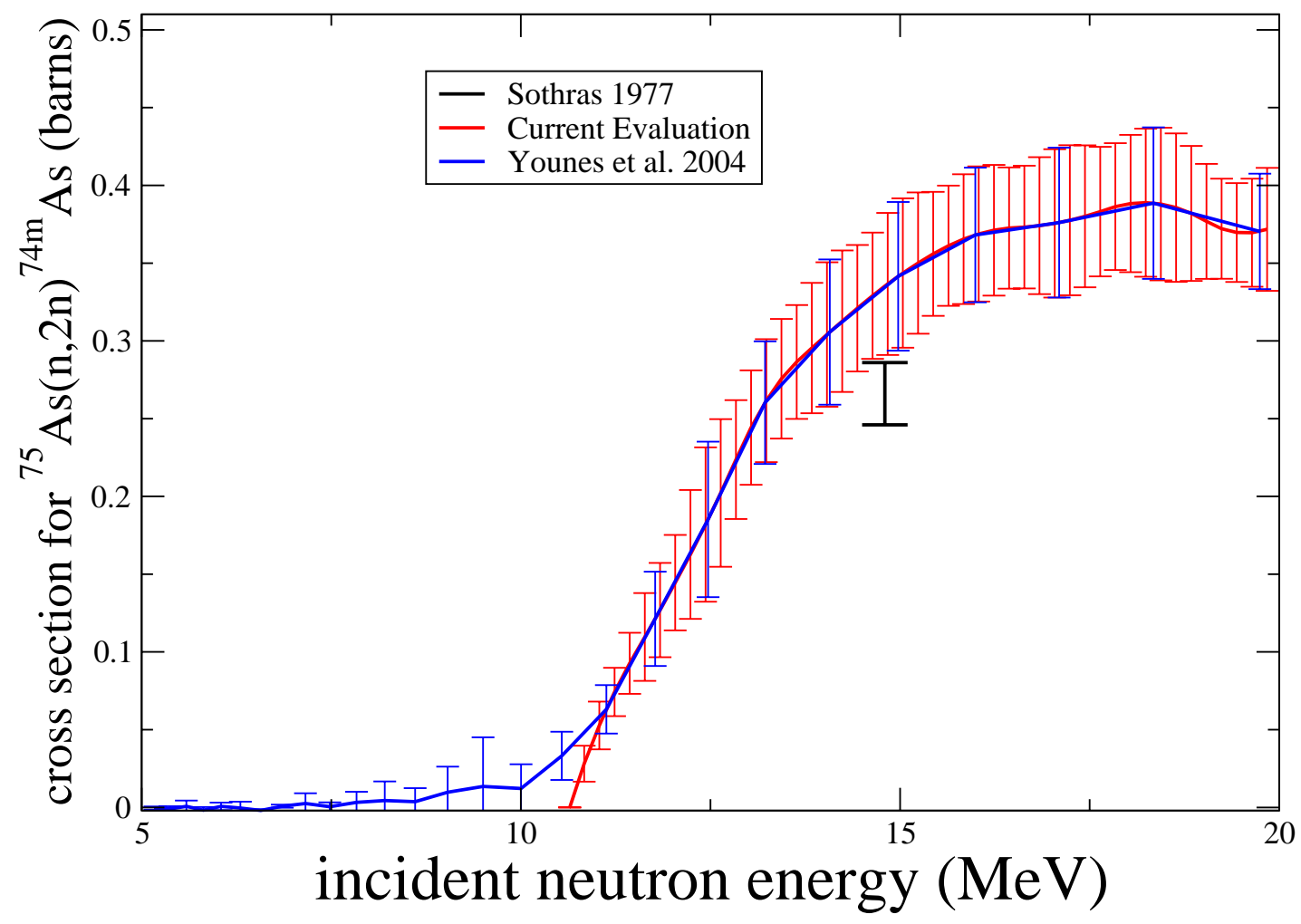

Fig. 2.- Data and the present evaluation for $(n, 2 n)$ production of the isomer in ${ }^{74}$ As. 


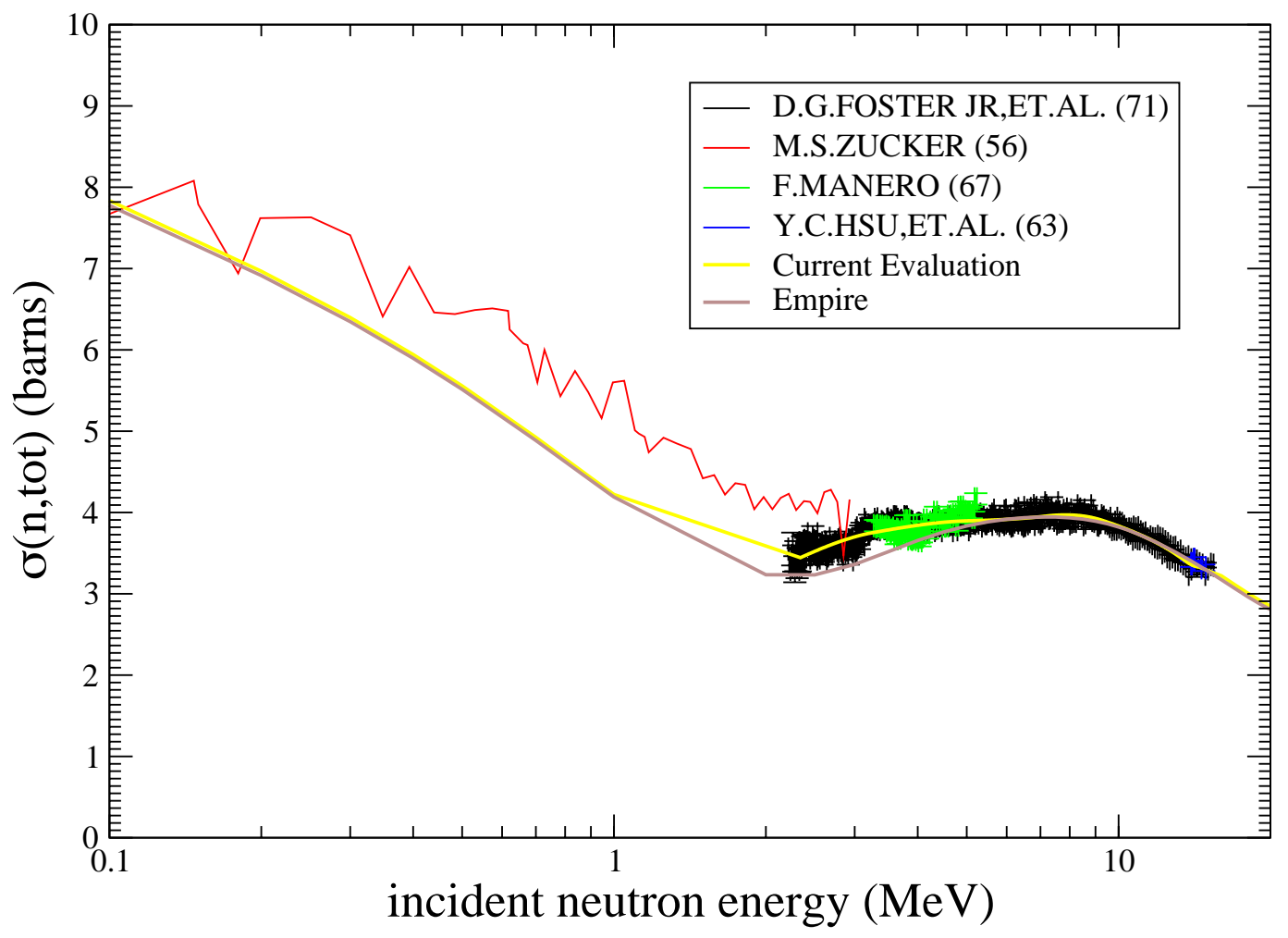

Fig. 3.- Experimental data, an EMPIRE calculation, and the current evaluation for ${ }^{75} \mathrm{As}$ (n, tot). Uncertainties in results of the 1956 Zucker experiment were not stated. This experiment was not used in our evaluation. 


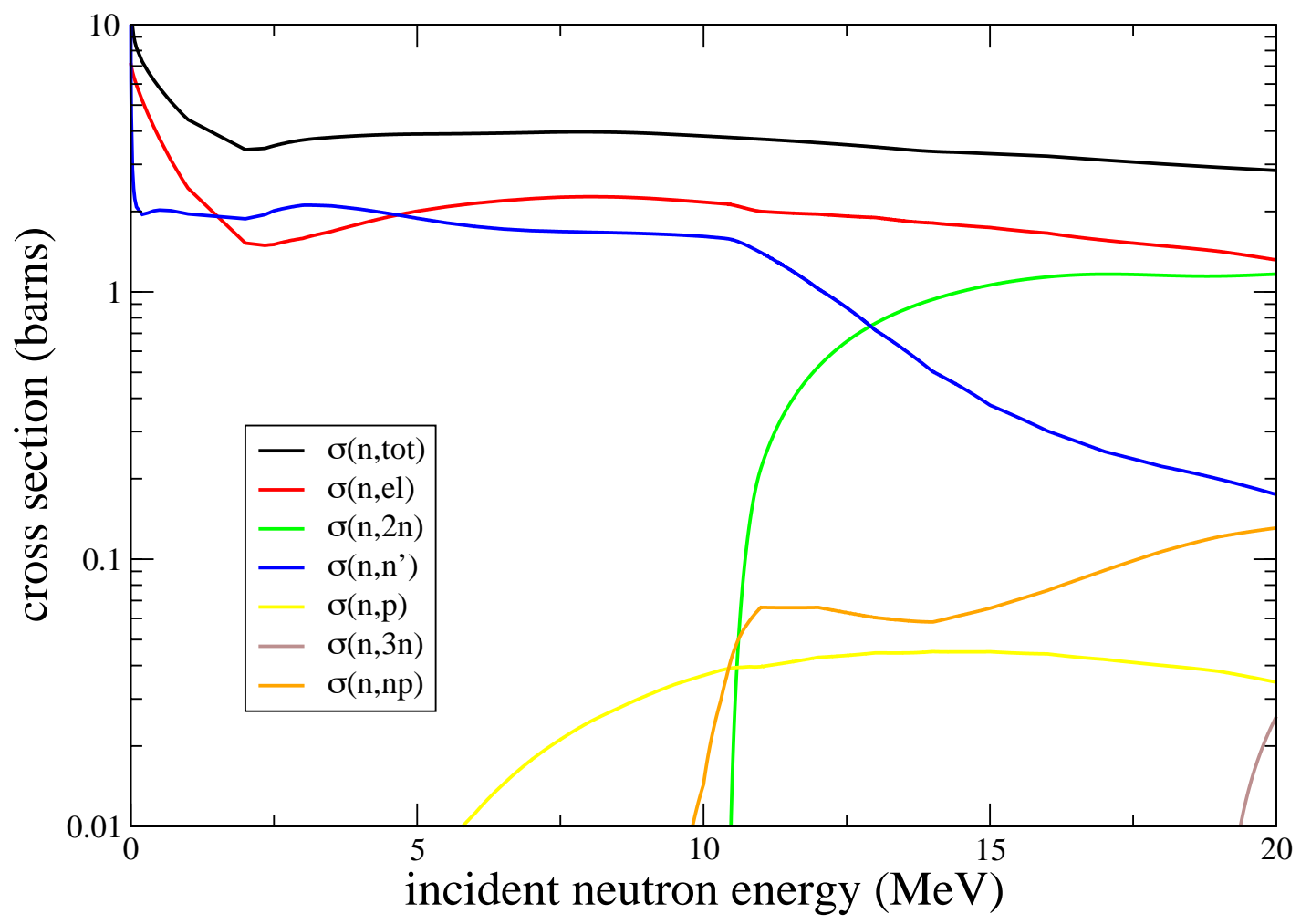

Fig. 4.- Our evaluations for cross sections describing some important $n+{ }^{75}$ As reactions. 


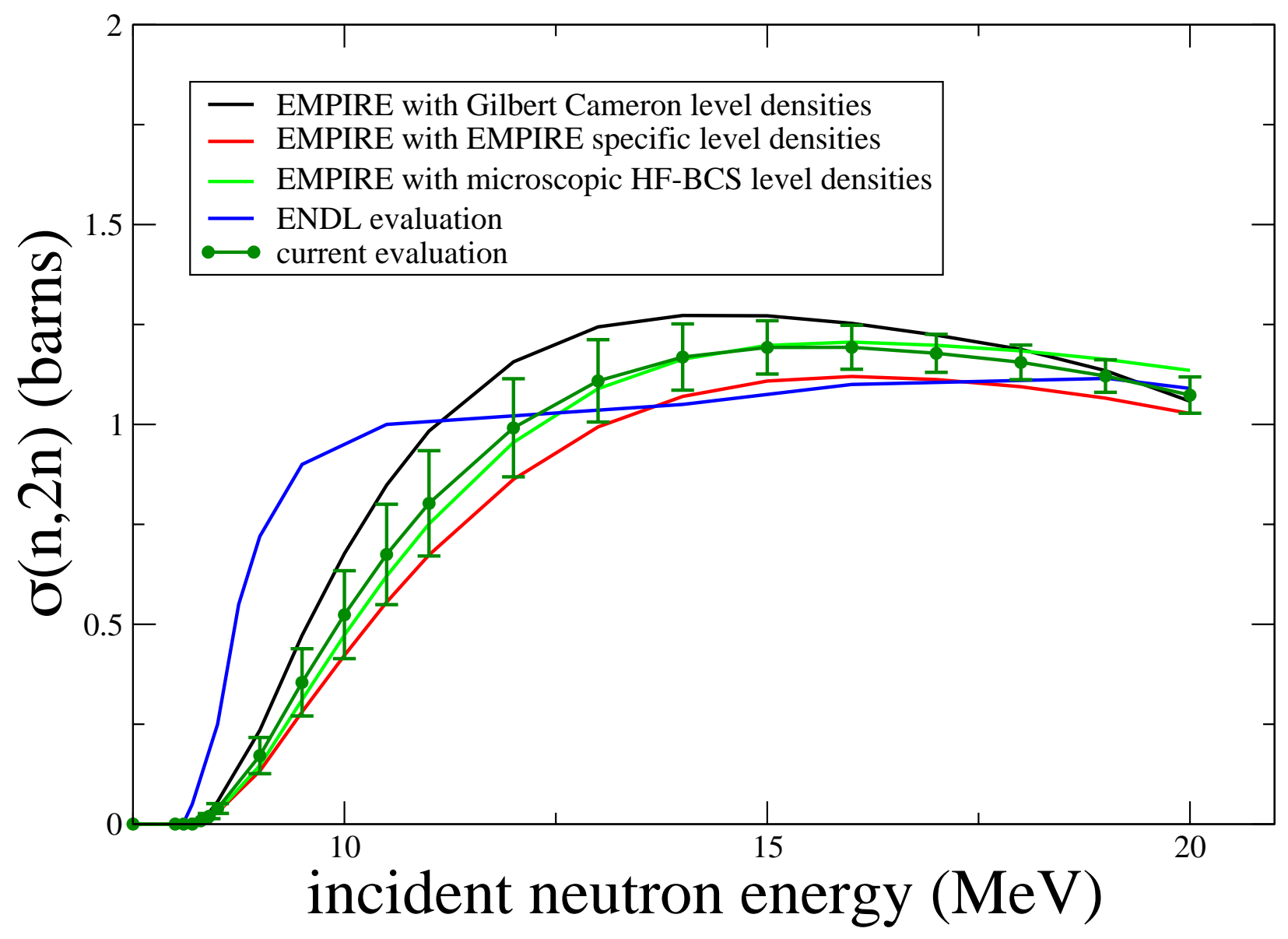

Fig. 5.- EMPIRE calculations, the ENDL evaluation, and the current evaluation for ${ }^{74 g} \operatorname{As}(\mathrm{n}, 2 \mathrm{n})$. The different EMPIRE calculations correspond to different parametrizations of the level density. See the text for a discussion of our current evaluation. 


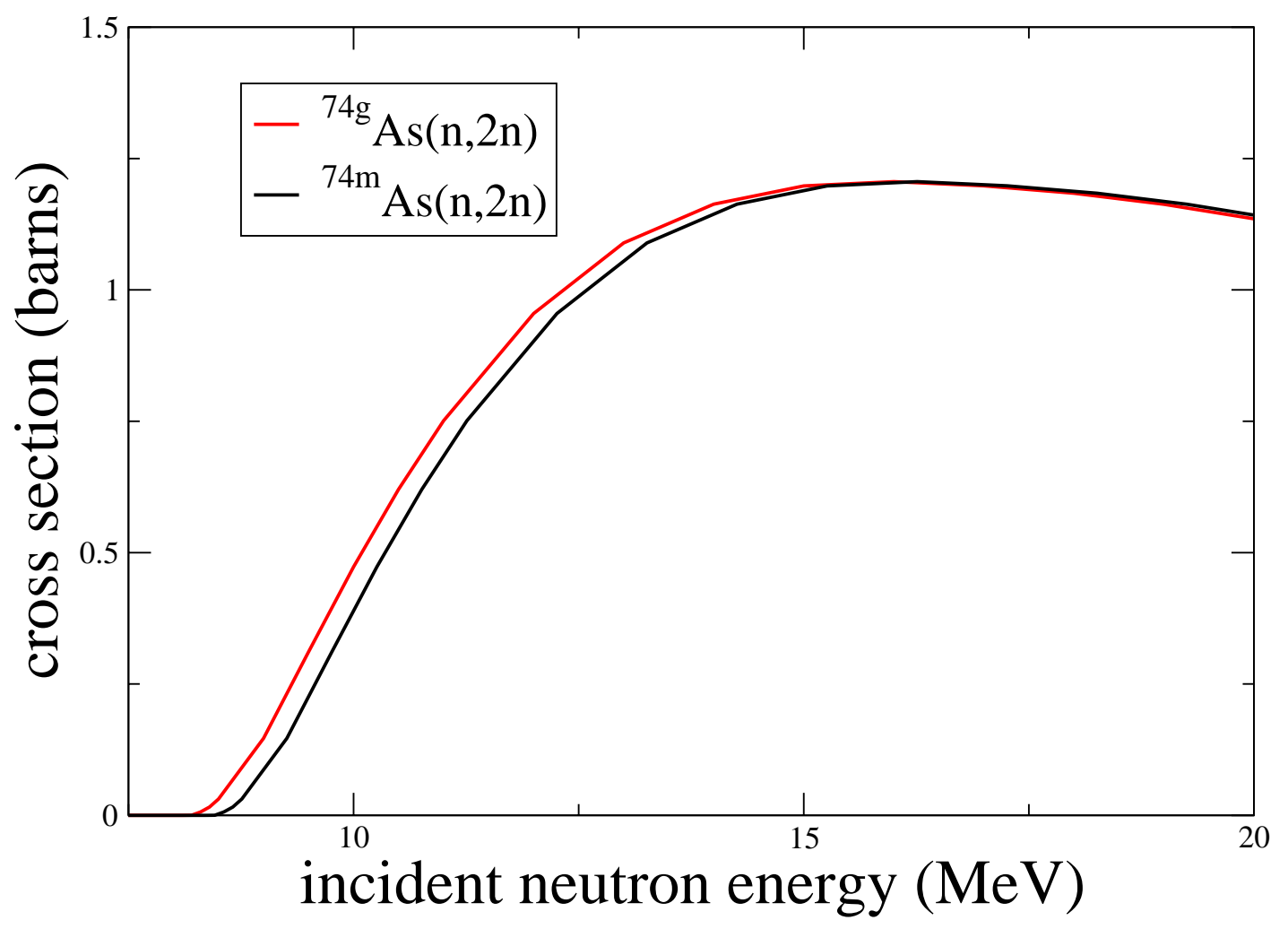

Fig. 6. - EMPIRE-based estimates for destruction of the ground and meta-stable states of ${ }^{74}$ As via the $(n, 2 n)$ reaction. For each calculation the Hartree-Fock BCS level density prescription was used (see figure 5). Note the approximate equality of the two cross sections. 


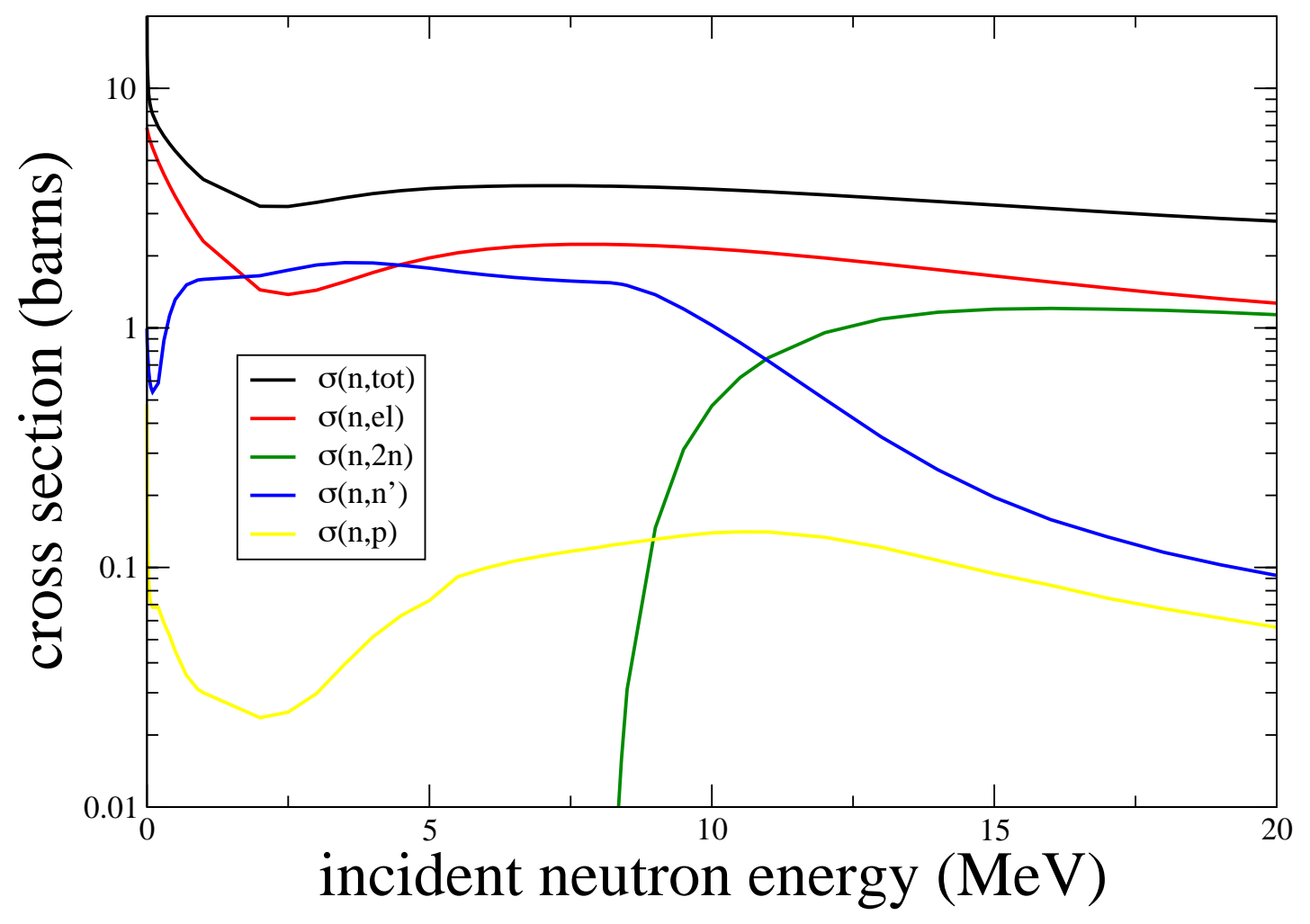

Fig. 7.- Our evaluation for cross sections describing some important $n+{ }^{74}$ As reactions. 


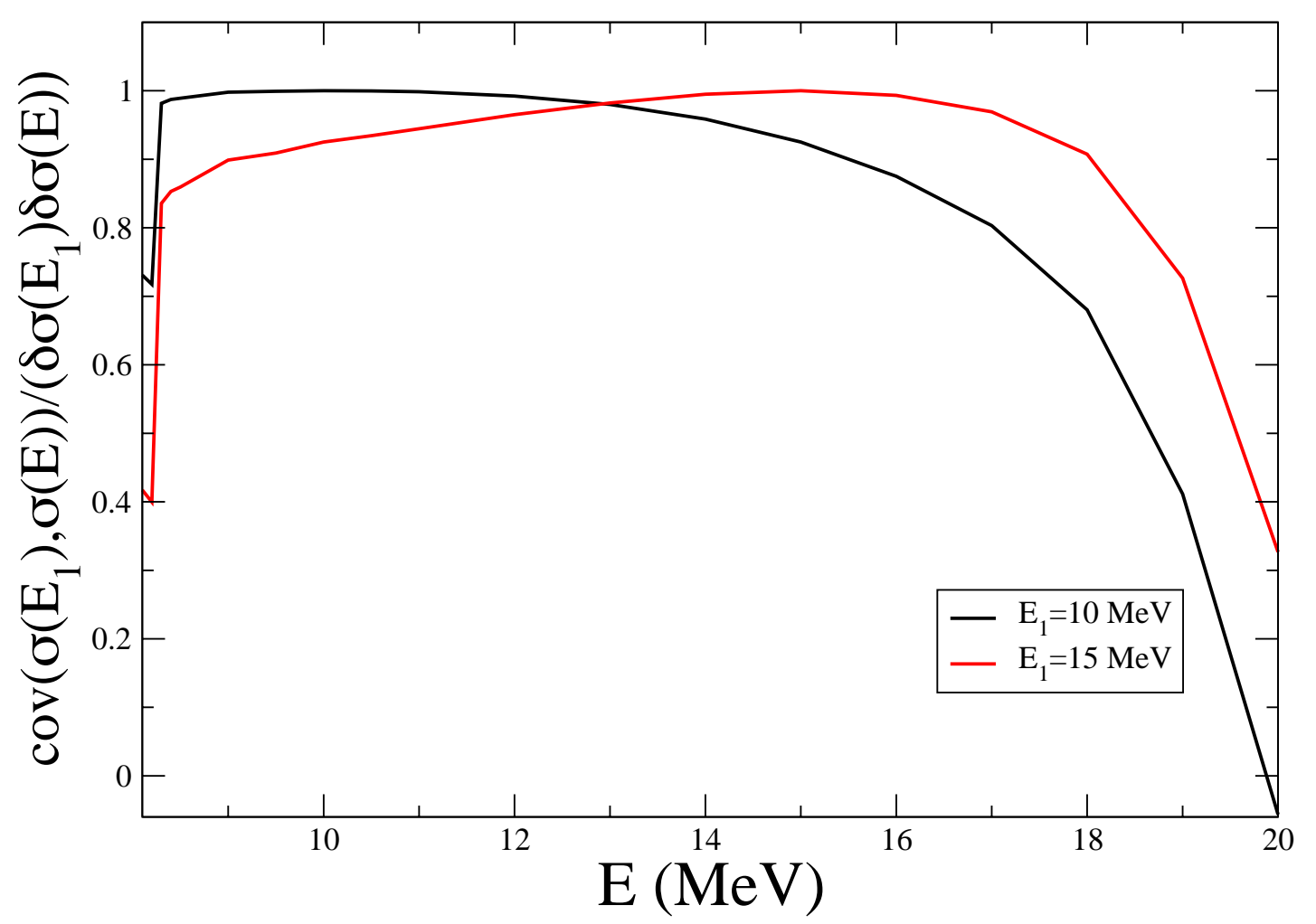

Fig. 8. - Estimates of the covariance matrix for the ${ }^{74} \mathrm{As}(\mathrm{n}, 2 \mathrm{n})$ reaction cross section. Note the strong positive correlations for incident neutron energies less than about $18 \mathrm{MeV}$. This reflects the tendency of different model calculations to have roughly the same shapes. At larger incident neutron energies the correlation tends to zero: changes in the cross section at $10 \mathrm{MeV}$ are approximately unrelated to changes in the cross section at $20 \mathrm{MeV}$. 